\title{
GLANDULAR FEVER: INFECTIOUS MONONUCLEOSIS
}

\author{
By Sir HENRY Tidy
}

E. Pfeiffer, a paediatrician of Vienna, gave, in I889, the first clear description of this disease under the title Drüsenfieber or glandular fever. He recognized that it was infectious and occurred in epidemics, described accurately the course of the enlargement of the cervical glands in young children, though he denied that other glands were involved, and stated that the glands never suppurated and that the prognosis was uniformly favourable. The blood was not mentioned.

Other observers in Germany soon reported epidemics. Spread of its knowledge was somewhat slow but Park West (1896) reported an epidemic in America and Dawson Williams (1897) one in England. The disease appeared to be successfully launched, but the diagnosis in sporadic cases rested on rather indefinite clinical features. It became confused with septic infections and rapidly fell into disfavour. By 1900 it was nearly dead. No mention of it appears in the "Medical History of World War I,' although subsequent information proves that it was not uncommon. Burnford (rgr8) is the single observer to whom the possibility occurred; he suggested that a group of cases with pyrexia and enlarged glands belonged to Pfeiffer's Drüsenfieber. Its memory was just kept alive by brief descriptions in general textbooks copied verbatim from book to book. It remained in suspended animation until 1920.

It is remarkable that during a period of 30 years, covering rapid developments in the knowledge of haematology, there should have been no systematic examination of the blood in a condition characterized by enlargement of lymphatic glands and often by enlargement of the spleen. To this lack of observation there is one exception. J. E. Burns (1908) fully described the lymphocytosis in two epidemics which he diagnosed as glandular fever, and published a well-documented report. The communication was overlooked until it was quoted by Bernstein (1940) in his monograph. Priority for the recognition of infectious mononucleosis clearly belongs to Burns.

Nevertheless the establishment of the haematological features of the disease did not come, for practical purposes, until 1920. Sprunt and Evans in November, 1920, published their observations on transient mononucleosis recorded in a series of six college students during the previous six years. They recognized that the condition was infectious, observed the general glandular enlargement and gave an accurate though brief description of the various types of cells in the blood. They were unaware of the existence of Pfeiffer's glandular fever and thought they had discovered a new diseasea very venial mistake-and named it 'infectious mononucleosis.' Downey and McKinley (1923) gave a complete description of the cells, beautifully illustrated, to which nothing effective has been subsequently added.

Meanwhile Morley and I, in June, 1920, had found a transient lymphocytosis in the case of a boy whom we diagnosed as suffering from glandular fever, and we reported it with other cases at a meeting of the Royal Society of Medicine in December, 1920.

The recognition of the identity of infectious mononucleosis and glandular fever was not immediate in America, though Longcope used both terms in 1922, but it was probably general by 1925. The material on which the observations were based in America was, and still is, pree dominantly from college students about the ages of 18 to 24 years, while in England it was mainly supplied by resident preparatory school boys of 8 to 14 years. Although any type of the disease may occur at any age, there are considerable differences, when numbers of individuals are involved, between the clinical manifestations in the two age-groups, the glandular enlargement being more marked at the younger ages. These factors no doubt account for the differences in nomenclature, for the disease is invariably known as infectious mononucleosis in America while in Britain it is generally named glandular fever.

Paul and Bunnell (1932) made the curious discovery that heterophil agglutinins to sheep's red cells are present in high titre in the blood in glandular fever and in no other known disease, a discovery which increased the interest in the condition.

Glandular fever commenced its clinical life as a simple, harmless adenitis. In 1922 the addition of the anginose type or monocytic angina widened the field. The epidemic of r930 added prolonged febrile forms. About 1935 the onset with jaundice was found to be not uncommon. The latest addition is the recognition of its association with neurological disturbances of a varied and often bizarre character but without permanent illeffects. One may wonder in what guise glandular fever will next make its appearance. 
Clinical Manifestations in Glandular Fever

' Juvenile' and 'Adolescent' Types

The onset in young children is usually acute. There may be a short prodromal period of malaise but this is commonly overlooked and the glandular enlargement often first attracts attention.

The cervical glands are most frequently involved. They tend to enlarge rapidly and may form a visible tumour within 24 hours. The mass is normally painless and only slightly tender, though young children may object to palpation. The gland which is most characteristically enlarged is deep to the sterno-mastoid muscle, about the middle of its length, and somewhat below the angle of the jaw; it lies transversely and can be felt on both sides of the muscle. There is usually some enlargement of axillary glands. Either here or at other sites this may be sufficient to constitute definite masses. Such a mass may be present without any involvement of the cervical groups.

In the fauces there is little change though some complaint of sore-throat is common. Eruptions are unusual. Torticollis is an occasional complication.

The temperature is raised to about $\mathrm{IOI}^{\circ}$ to $103^{\circ}$. Constitutional symptoms are slight and the patient does not appear ill. The spleen becomes palpable in about half the cases, but only one or two finger-breadths. The main mass of the glands and the constitutional disturbances generally subside in the course of a few days, and the patient has recovered in one to two weeks.

Suppuration of the glands is no part of the ordinary course of glandular fever but in very rare cases has followed a pre-existing tonsillitis.

The glands, although much smaller, may remain palpable for many weeks or even several months.

Mononucleosis is often, but not invariably, present when the glands appear, otherwise it develops within a few days; not infrequently this is the date of the first examination. The total leucocytes in most cases lies between 10,000 and 20,000 per cm. with 60 to 80 per cent. of mononuclear cells. Both monocytes and lymphocytes contribute to the excess, but the monocytes subside rapidly and leave the lymphocytes to predominate. Some degree of lymphocytosis may linger for several months.

Recrudescences or relapses are far more frequent than is usually believed. The course may be similar to the original attack but tends to be milder. Recurrences may take place after considerable periods of freedom; there may be several over a period of some months, gradually decreasing in severity. I have been told of cases in which they extended over one or more years but I have not seen them, and the diagnosis should te carefully reviewed.
The incubation period is between five and 1 ? days in the great majority of cases, but in spite of many studies it is impossible to fix an upper limito

The description given above applies especially: to the type which is seen in children between the् ages of 5 and 15 or 16 years, sometimes referred to as the 'juvenile form.' Under the age of years the picture is less characteristic; the con̄ stitutional symptoms are often more severe an the glandular enlargement slighter, and the diagnosis may be difficult.

Among adolescents and young adults the course tends to be longer and more insidious and the glandular enlargement less marked than in the్ juvenile form. The onset is slow with fatigue and depressed health, gradually becoming sufficient to cause the subject to report to a medical officeriv The glands may be found then or subsequentlyos often on routine examination. This is the format in which glandular fever is commonly seen in America among college students, and Americas descriptions of the disease are essentially based on? this age-group. They have few resident 'pre paratory schools' as we know them and cons sequently their clinicians have only a limited ex perience of the juvenile type.

As mentioned above, any superficial glamds may enlarge; this includes the pre-auricular, si़्ञ maxillary, submental, supra-trochclear and cipital glands. There is also evidence that deep glands may be involved. I have seen cases in which pressure on the bronchi has produced transient obstruction with very confusing physica? signs. This complication has been confirmed radiologically by Nelken (1926). Abdominal pain may be severe and due to enlargement of deep glands.

\section{Anginose Type, Monocytic Angina}

Neither resident college life nor resident pre paratory schools are a part of the educationab system in Europe outside Britain, and the recogin nition on the Continent of a recoverable mono nucleosis (other than that of whooping cough) was initially based on a different type of materiap appearing in entirely other guise from the glandular forms presenting in America andE. England.

Deussing, in t9r8, reported a series of cases with transient absolute lymphocytosis under the title 'Ueber diphtherieähnlicher Anginen mitu lymphatischer Reaction' (angina resembling diph theria with a lymphatic reaction). But it was communication by Schultz in $\mathbf{1 9 2 2}$, to the Congress for Internal Medicine, on "Monozytenangina :" which first attracted attention to the subject. Both communications were based on patientso admitted to a hospital for communicable diseases? 
with membranous tonsillitis in which no diphtheria bacilli were found and in whom recovery followed without the injection of antitoxin.

The Continental authorities went astray from the start. They were obsessed with the idea that the development of the lymphocytosis was due to a constitutional peculiarity of the patient resulting in a 'lymphatic reaction' and that the same angina in other individuals would result in a polynucleosis. The possibility of infection was scarcely considered. Secondly, they embarked on a tedious and sterile dispute amongst themselves covering many years as to whether the cells were monocytes or lymphocytes. They failed to recognize that both types of cells were often present at the same time and that either type might predominate at different periods in the same patient. Not until Glanzmann's monograph in 1930 did they admit that monocytic angina was a manifestation of infectious mononucleosis and recognize the influence of an infective factor as opposed to a constitutional diathesis.

The definite differentiation of monocytic angina from diphtheria was a clinical advance of importance, and it is surprising that so little notice has been taken of it in American and British literature. It is rarely.properly described in textbooks under the headings of either tonsillitis or diphtheria or glandular fever, and its existence is not yet generally recognized by the profession. Even nowadays it is frequently diagnosed as acute tonsillitis, treated with sulphonamides or penicillin and the favourable course ascribed to the therapy. Yet it is a condition of considerable severity and is proof of the presence of glandular fever.

Monocytic angina or the anginose type of glandular fever is characterized by the development of a tonsillar membrane or of ulceration. The membrane in typical cases is indistinguishable in appearance from that of diphtheria, although it is a true membrane, and it often forms very rapidly. It never involves the larynx. Oedema of the neck and tenderness of the enlarged cervical glands is common, but not to the extreme degree of diphtheritic bullneck, and suppuration is very rare. Oedema of the fauces causes great discomfort and anxiety, but in spite of this and the high temperature, often about $104^{\circ}$, the patient does not appear to be toxic nor does he become so, although the membrane may persist for several days or more than a week before separating, after which the symptoms improve with surprising rapidity. There has sometimes been a previous period of slight malaise for two or three weeks with increasing sore throat, and some glandular enlargement may or may not have been observed.

Mononucleosis is generally present at the time of appearance of the membrane and is always present and absolute within a day or two, but at the onset it is often only relative and an initial leucopenia is common. It is possible that the angina is a complication following a mild attack of glandular fever two or three years previously. The development may be the result of the leucopenia which is often present initially or of the mononucleosis. There is no proof that the disease is infectious at this stage or that it spreads in this form.

\section{Prolonged Febrile Types}

An extensive epidemic in England in 1930 was characterized by the severity and long duration of the attacks and by the high proportion occurring in adults. It apparently represents the full development of glandular fever. Sporadic cases and milder grades are fairly common but this is the only recorded occurrence of the type in epidemic form (Tidy, 1931).

At the beginning the diagnosis of typhoid was often made in spite of the uniformly negative agglutinations and it was some time before the epidemic was recognized as glandular fever. Indeed the identity could scarcely have been suspected before the discovery of the changes in the blood.

The epidemic exhibited clearly that in severe forms the characteristic features of glandular eno largement and mononucleosis develop late and the glands rarely attain any great size. In many cases the severe constitutional disturbances are already subsiding before the glands become palpable.

The initial febrile period is of variable duration, and recrudescences may lengthen it to many weeks. During this stage there may be no diagnostic features either on physical or laboratory investigation. Headache is often severe. There may be a few rigors. Sweats are nearly always profuse; they are greatly increased by the administration of antipyretics and then become associated with extreme exhaustion. Wassermann reaction and Kahn's test may become temporarily positive.

An eruption is common. The type varies but it may be noted here that it is sometimes indistinguishable from typhoid.

The temperature has no regular course but it has generally fallen to normal or nearly normal before the glands enlarge, when it rises again. The glands are often discovered by chance or by a routine examination when the temperature rises. The spleen may become palpable at this stage. Rapid improvement often follows and convalescence sets in, but recurrences are not uncommon.

The course of the blood changes is important. At the onset there may be a definite polynucleosis; this often misleads observers who are unaware of 
the possibility. The polynuclear cells always fall and for a period the blood count may be strictly within normal limits. Leucopenia is often present about the time the glands commence to enlarge. In some cases there is a leucopenia at the onset, but the febrile period in these is rarely prolonged; the earlier stage seems to have been cut off so to speak. The glandular enlargement is always associated with mononucleosis.

Four groups of clinical manifestations have so far here been indicated: (I) Pfeiffer's glandular fever, in the 'juvenile' form usually seen in children, characterized by rapid and visible swelling of the cervical glands and a short duration, (2) infectious mononucleosis in young males with a longer but milder febrile stage and comparatively slight glandular swelling, (3) the anginose type or 'monocytic angina,' and (4) febrile types with late and slight enlargement of glands.

This grouping was useful while the clinical features of the disease were being carefully studied, but the disease is a single entity and every permutation and combination of the four groups occur.

\section{Eruptions}

The rash in glandular fever may cause considerable difficulty owing to its close simulation of many of the exanthemata.

During the course of glandular and anginose types well-defined eruptions are rare and tend to be transient. In the febrile forms eruptions are commoner, though the incidence has varied greatly in different epidemics; the rash usually appears within a few days of the onset but may develop at any point in the course.

There are two common types: (a) macular or maculo-papular; this is the eruption most frequently seen in the febrile forms and may closely resemble the rash of typhoid, and (b) rubelliform; this is the commoner type in the glandular forms and may be indistinguishable from rubella. It is of interest that I have on several occasions known a rubelliform eruption appear and subside a week or two before the development of an attack of glandular fever, an initial diagnosis of rubella having been made without hesitation. I have also been told on good evidence of transient eruptions a week or two before the development of the membrane in the angiose form.

Cases of glandular fever have been admitted to infectious disease hospitals with rashes resembling typhus, measles, scarlet fever, chicken-pox, erythema nodosum and urticaria. I have never personally seen an eruption resembling fullydeveloped chicken-pox.
The Blood Picture

It is probable that mononucleosis develops in every case of glandular fever.

All the blood-forming tissues are affected $\overrightarrow{\vec{F}}$ myeloid, monocytic (or reticulo-endothelial) an of lymphoid, but at different times and to different degrees and varying in different cases and $\overline{\bar{n}}$ indeed, in the same case at different stages. The effect on one system may be decreasing while on? another it is increasing thus producing the rapides changes in the blood picture which are so charac- $-\overrightarrow{0}$ teristic of the disease. The full sequence of thechanges is best observed in the long severe febrilew cases but unfortunately the diagnosis is rarelyo made in their early stages.

The myeloid system is earliest involved but less 3 . constantly or severely and for a shorter time than the other systems. In mild cases there may be noj change in the circulating myeloid cells, but in severer forms an initial polynucleosis, such as I 5,000 to 20,000 leucocytes per $\mathrm{cm}$. with 75 per? cent. polynuclears is not infrequent. The pos $-\vec{c}$ sibility of such polynucleosis is not generally realized and frequently causes an error in

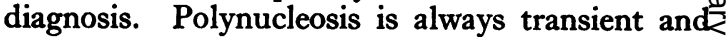
initial and never develops during the course of the attack. The rise of the mononuclear reaction mor overlap the fall of the polynuclear cells, but in the more severe forms this reaction is delayed and theo blood count becomes within normal limits ands may remain so for two to three weeks or more, oro may fall further to a leucopenia before the mono-0 nucleosis appears. Leucopenia also is fairly common at the onset; it is mainly due to granu- $\overrightarrow{\overrightarrow{0}}$ lopenia but even the lymphocytes may fall. In 3 milder clinical types, such as the ordinary? glandular forms, a mononuclear reaction may be present at the first examination or within a fewo days of onset.

The monocytic and lymphocytic reactions over-음 lap but the monocytic system is the first to subside and a pure lymphocytosis is finally left. Soo rapidly may alterations take place in the types of white cells and their number and so great are the differences in different cases that no single blood $\supset$ picture is exclusively typical of the disease. But? most characteristic during the active stages is then presence simultaneously of various types of mononuclear cells, particularly with a high incidence of 0 monocytes, and the rapidity with which the relative ${ }^{N}$ proportions change.

The total number of leucocytes does not rise too very high figures. About 10,000 to 15,000 per $\mathrm{cm}$. is common, over 20,000 is unusual and over? 30,000 is rare. The limit is about 50,000 but somewhat higher numbers have been recorded in웅 infants.

The percentage of mononuclear cells is gener- $\mathbb{D}$ 
ally between 60 and 70 when the disease is fully developed, over 80 per cent. is rare and over $9 \circ$ per cent. very rare, but there are authentic instances and I have seen it several times.

The existence of a specific 'infectious mononucleosis cell '. is in dispute. American authorities of standing have described them minutely. British authorities, such as Whitby and Britton, believe that they are primitive monocytes, an opinion with which I agree. It would be unsafe to base a diagnosis on the presence of such cells and certainly unsafe to exclude glandular fever on their absence, since as the attack subsides the surviving cell is undoubtedly a lymphocyte, large or small.

\section{Heterophil Antibodies, Paul-Bunnell Reaction}

The term heterophil antibodies is applied to antibodies which can react with certain antigens which are unrelated to the cause of their development. Here it is a question of the development in human serum of antibodies which agglutinate sheep's red cells. Such antibodies are present in low titre in normal serum and similar antibodies may be present to a higher titre in serum sickness or after injections of horse serum. Paul and Bunnell in 1932 discovered the curious fact that such antibodies develop in high titre in human serum in glandular fever, and in no other disease, with some academic exceptions. Though similar, the three types of agglutinins are not identical and they can be separated by appropriate absorption tests.

The technique of the reaction unfortunately has not been standardized. Confusion has arisen in America over the method of recording the dilution of serum, but this does not appear to have happened in Britain. With a simple technique such as that described by Smeall (1942) a titre of $1 / 64$ is almost proof of glandular fever and a titre of $1 / 128$ is absolute proof. If there is any doubt when the titre is $1 / 64$, the test can be repeated or absorption tests performed.

Important questions which arise are at what stage the reaction becomes positive, in what proportion of cases it is positive, and whether or not a negative reaction excludes glandular fever.

In the ordinary mild types the reaction is frequently positive at the first examination, which may be four or five days after the onset. If the examination is earlier the test may be negative or indefinite, the titre rising in the next few days. Owing to the certainty with which the diagnosis can often be made on clinical and haematological grounds, the test is not always repeated. Nevertheless in these circumstances the reaction is positive in nearly 90 per cent.

But in the severer febrile forms the reaction may and generally does remain negative during the weeks of pyrexia and constitutional disturbances, and becomes positive about the same time as the mononucleosis and glandular swelling develop. $\stackrel{\varrho}{c}$ It is striking how often the constitutional symptoms rapidly abate within a few days of the rise in titre. Thus a positive reaction is related to the $\overline{0}$ end of an attack rather than to the beginning, and $\overline{ }$ it may well be connected with the development of $\bar{m}$ immunity as Himsworth (1940) suggested. On $\stackrel{\circ}{\circ}$ more than one occasion I have known the reaction become positive for the first time during a relapse, o but I have also known relapses take place while the titre is still high.

It is very important that this time-relationship should be borne in mind. The development of agglutinins appears to be associated with or related to the stage of glandular enlargement and mononucleosis. A negative reaction during the stage of constitutional symptoms is therefore not surprising and must not be interpreted as ex- 6 cluding the possibility of glandular fever. The 은 agglutination reaction is of most value in confirming that enlarged glands and mononucleosis are due to glandular fever.

The titre has no constant relationship to the severity of the disease, the extent of the glandular swelling or to the degree of mononucleosis. The time during which a reaction remains positive is very variable, but little more is known. The titre may rise to a very high dilution and fall again. to negative in the course of a few days. In ordinary mild cases it usually becomes negative within two weeks of recognition, but it may persist for several weeks and has been found still positive after several months.

The significance of a negative test specially arises in epidemics in which all cases are reported as negative, and in sporadic cases in which the test is repeatedly negative. The question arises whether or not there are two types of glandular fever giving respectively positive and negative reactions. There is nothing inherently improbable in the existence of two viruses, but until we know more about heterophil agglutination the evidence must be regarded as inconclusive.

I agree with the opinion of Paul and others that a positive reaction is proof of glandular fever and a negative reaction does not exclude it.

\section{Neurological Manifestations}

The neurological manifestations of glandular fever have attracted attention recently and a number of cases have been recorded in the last few years (Geliebter, 1946; Schneider, 1947). The presence of glandular fever in similar cases must previously have been overlooked. The existence of encephalitis was suspected by Longcope (1922) and by Glanzmann (1930), but the first clear descrip- 
tions of neurological features were given by Epstein and Dameshek (1931) and by Johannsen (t93r). The clinical pictures are extraordinarily varied and bizarre, and no two cases appear to be quite similar. The brain (encephalitis), meninges, cord, cranial nerves and peripheral nerves may be affected either separately or in combinations or sequences. There is no constant order in which the ordinary manifestations of glandular fever and the neurological symptoms respectively develop, or in their comparative severity. Thus the glandular enlargement, lymphocytosis in the blood and in the cerebrospinal fluid, and meningeal or other neurological symptoms may develop and subside simultaneously as in Epstein and Dameshek's case. In other cases the glandular fever may run its course and subside to be followed by nervous symptoms, and lymphocytosis in the cerebrospinal fluid, but with a normal blood picture and agglutination titre. Or again the symptoms of a benign lymphocytic meningitis may be subsiding before the features of glandular fever appear. In this last group the blood count may show an initial polynucleosis even with a high mononucleosis in the cerebrospinal fluid. It is noteworthy that in the more severe neurological forms the glandular swelling tends to be slight as with other severe types of glandular fever and may be overlooked during convalescence from the nervous features unless specifically sought.

The interpretation of the Paul-Bunnell reaction in neurological cases needs careful consideration. The heterophil agglutinins have been estimated in all recorded cases since 1938 , and the test always has been positive in the blood at some stage with the exception of two doubtful cases in sisters (Thelander and Shaw, 194I), but not in the cerebrospinal fluid. This may only mean that the diagnosis of glandular fever is not made unless the reaction is positive.

In assessing the value of a negative reaction the time-relations described above must be taken into consideration. Cases of benign lymphocytic meningitis have been recorded in the neurological literature in which glandular fever is held to be finally excluded by a negative agglutination, although the reaction has been performed at a point during the course when it would not be expected to be positive. Neurologists have not entirely familiarized themselves with the protean manifestations of glandular fever and the timerelations of the development of agglutinins.

The symptoms of benign lymphocytic meningitis are exactly reproduced in certain of the cases. There are, of course, a number of infections and viruses which are known to produce this syndrome, and it is not suggested that more than a proportion is due to glandular fever. But some undoubtedly are, and the association with glandular fever is 3

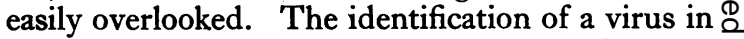
a case of benign lymphocytic meningitis does not $\subseteq$ exclude the possibility.

Recovery from neurological manifestations $\operatorname{can} \frac{\overrightarrow{+}}{\frac{\vec{O}}{O}}$ take place with extraordinary rapidity. A coma- 듬 tose and paralysed patient with an extensor plantar response may be apparently normal $\bar{\Phi}$ mentally and physically in three days. The question of encephalitis requires further observa- $\infty$ tion. Severe headache is the commonest symp- $\overrightarrow{0}$ tom in neurological cases and is also an occasional complaint in ordinary types. It is often observed $\vec{\omega}$ that children and adolescents may take a sur- $\frac{\mathrm{O}}{0}$ prisingly long time, six to 12 months, to re- $\frac{0}{3}$ cover their previous powers of concentration and application after a simple attack, and it is possible o that this is a sequel of encephalitis.

\section{Association with Jaundice}

Jaundice is now not uncommon at the onset or $\rightarrow$ during the course of the severer forms but the association has become frequent only in the last 15 years. It was not recorded in the epidemic in England in 1930, but in 1935 and 1936 many cases were observed in St. Thomas's Hospital and $\overrightarrow{0}$ it is now a recognized complication. Whe occurring at the onset the jaundice is often considerable severity and there is nothing to dis tinguish the clinical condition from an ordinary infective hepatitis. As the jaundice subsides the pyrexia persists and the diagnosis of glandular $\stackrel{\mathbb{Q}}{\varrho}$

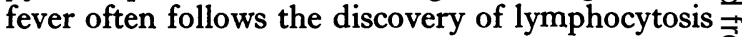
in a routine blood count or the observation of some glandular swelling.

Jaundice adds the symptoms of infective hepatitis to the symptoms of glandular fever but does not appear otherwise to affect the course. Jaundice is rarely severe when developing later in the illness. Whether or not the jaundice is due to a separate virus cannot at present be determined. $\dot{\delta}$

\section{Prognosis}

I have personally never seen a case of glandular $\vec{D}$ fever in which recovery was not complete. Nevertheless there are exceptions. Several of the small number of recorded deaths are open to doubt, but ${ }^{\circ}$ a few are authentic. In some of these there has $N$ been an extraneous factor such as pre-existing $N_{\omega}^{N}$ sepsis or debility in children. A patient has died in the comatous stage of a neurological complica-o tion. But it can be accepted that the ordinary forms are devoid of danger. The long period of $\stackrel{\odot}{+}$ partial debility and lack of concentration which 0 may follow even mild attacks especially in children has been referred to above. This was formerly $\stackrel{\odot}{\Phi}$ ascribed to an effect on the blood but there is no $\stackrel{\mathbb{Q}}{\mathscr{Q}}$ 
evidence of resulting anaemia and it may be due to encephalitis.

\section{Prophylaxis}

The degree of infectivity is not great and second cases in a family are unusual. On the other hand, susceptibility appears to be almost universal. Consequently, if two or three cases develop in a school and there is an opportunity for mass infection all the other children may become infected.

- It is extremely difficult to be dogmatic about the duration of infectivity. It is probable that the disease is infectious only in its early stages but the possibility of infectivity during relapses cannot be excluded. It is reasonably certain that the anginose type is non-contagious.

Isolation should be carried out in accordance with the dictates of common sense for a disease which is undoubtedly infectious but only of a moderate degree of infectivity and with a favourable prognosis. A reasonable course is to isolate a case for one week after the mass of the glands has subsided and the temperature has become normal.

\section{Diagnosis}

Possible errors in diagnosis are numerous and mistakes in practice are not uncommon, but in ordinary attacks they usually settle themselves without important consequences. They will not be considered seriatim or fully but the following brief points may be noted.

The distinction from mumps is decided by the fact that the parotid gland is never affected. The differential diagnosis from sepsis may be difficult; the cases in which $I$ have been in serious doubt have nearly always proved to be sepsis, and it is advisable to treat them as such. Vincent's angina never causes lymphocytosis. From rubella the rash may be indistinguishable.

In the severer febrile forms there may be no means, either clinical or pathological, of establishing the diagnosis for several weeks. This may also apply to onset with jaundice or with neurological symptoms. The correct interpretation of a negative agglutination reaction is important. In the blood the most characteristic features are the simultaneous presence' of numerous types of mononuclear cells, and increased number of monocytes normal or slightly abnormal, and the rapidity with which the differential count changes. Difficulties may arise from the initial polynucleosis or initial leucopenia or from the almost normal blood count which may be temporarily present in the course of the long febrile forms. The blood changes should not cause difficulty in differentiation from chronic leukaemia. In acute leukaemia the toxic symptoms are always severe.

An occasional but extremely difficult diagnosis may be caused by the rare, slowly-progressive chronic lymphoid leukaemia. In the earlier stages there are periods of exacerbation with pyrexia and moderate glandular swelling. The lymphocytes for several years may be only at the upper limits of normal. They gradually creep up and the diagnosis long suspected slowly becomes confirmed. Diagnosis is specially difficult when it is asked for on a patient some months after a pyrexial attack with lymphocytosis considered at the time to be glandular fever. Either lymphocytosis or glandular swelling may persist for several months after glandular fever, but if both features are present for six months the diagnosis must be considered to be in doubt, unless it has been fully established. I have watched three such cases, originally diagnosed doubtfully as glandular fever, which gradually developed into fatal lymphoid leukaemia or lymphosarcoma over periods of three to ten years.

\section{Treatment}

Many drugs have been claimed to abort attacks and one by one have been discarded. I have found no difference between those so treated and those untreated. Sulphonamides are valueless? and their use should be deprecated in a condition in which the blood-forming tissues are in sensitive state. Antipyretics are contraindicated in severe febrile cases as they aggravate the sweating and increase prostration. Penicillin is indicated in the anginose type and there is evidence of its value; it has no influence on the course of other types. I have no experience of chloromycetin or aureomycin.

\section{BIBLIOGRAPHY}

BERNSTEIN, A. (1940), Medicine, 19, 45.

BURNFORD, J. (1918), Brit. Med. $\boldsymbol{f}, 2^{2}, 230$.

BURNS, J. E. (1909), Arch. Intern. Med., 4 , 118.

DOWNEY, H., and MCKINLAY, C. A. (1923), Arch. Intern. Med., EPSTEIN, S. H., and DAMESHEK, W. (1931), New England $\mathcal{Y}$. EPSTEIN, S. H., and
Med., 205, 1238 .

GELIEBTER, S. (1946), Lancet, 2, 753.

GLANZMANN, E. (1930), 'Das Lymphaemoide Drusenfieber,' Berlin.

HIMSWORTH, H. P. (1940), Lancet, $1,1082$.

JOHANNSEN, A. H. (I93I), Acta Med. Scand., 76, 269.

LONGCOPE, W. T. (1922), Amer. F. Med. Sci., 164, 781.

NELKEN, C. (1926), Monatschr. $f$. Kinderh., 31, 154.

PAUL, J. R., and BUNNELL, W. W. (1932), Amer. F. Med. Sci., 183,90.

PFEIFFER, E. (1889), fahrb. f. Kinderh., 29, 257.

SCHNEIDER, T., and MICHELSON, D. A. (1947), South African Med. F., January, 1947.

SCHULTZ,' W. (1922), Deut. Med. Wochenschr., 48, 149 r.

SMEALL, J. T. (1942), Edin. Med. F., 49, 29 I.

SPRUNT, T. P., and EVANS, F. Ä. (1920), Bull. Fohns Hopk. Hosp., 3r, 4 IOO.

THELANDER, H. E., and SHAW, E. B. (1941), Amer. F. Dis. Child., 61, i $13 \mathrm{r}$.

TIDY, H.' L., and MORLEY, E. B. (1921), Brit. Med. f., r, 100.

TIDY, H. L.' (1931), Proc. Roy. Soc., Med., 25, 155.

TIDY, H. L. (1934), Lancet, 2,180 .

TIDY, H. L. (1946), Laniet, $2,819$.

WEST, J. P. (1896), Arch. Pediat., 13, 889.

WILLIAMS, D. (1897), Lancet, 1, 160. 